\title{
Modelling Interacting Epidemics in Overlapping Populations
}

\author{
Marily Nika ${ }^{1}$, Dieter Fiems ${ }^{2}$, Koen de Turck ${ }^{2}$, and William J. Knottenbelt ${ }^{1}$ \\ 1 Department of Computing, Imperial College London, London SW7 2AZ, United \\ Kingdom, \\ Email: $\{$ marily,wjk\}@doc.ic.ac.uk \\ ${ }^{2}$ Ghent University, Department TELIN, St-Pietersnieuwstraat 41, 9000 Gent, \\ Belgium \\ Email: \{dieter.fiems,kdeturck\}@telin.ugent.be
}

\begin{abstract}
Epidemic modelling is fundamental to our understanding of biological, social and technological spreading phenomena. As conceptual frameworks for epidemiology advance, it is important they are able to elucidate empirically-observed dynamic feedback phenomena involving interactions amongst pathogenic agents in the form of syndemic and counter-syndemic effects. In this paper we model the dynamics of two types of epidemics with syndemic and counter-syndemic interaction effects in multiple possibly-overlapping populations. We derive a Markov model whose fluid limit reduces to a set of coupled SIR-type ODEs. Its numerical solution reveals some interesting multimodal behaviours, as shown in our case studies.
\end{abstract}

Keywords: Epidemics, Social Networks, Syndemic, Counter-syndemic

\section{Introduction}

You think because you understand 'one' you must also understand 'two',

because one and one make two. But you must also understand 'and'...

Rumi (13th century Persian Poet)

Epidemics of various kinds have been an important focus of study throughout human history. As health care standards have risen and information technology has advanced over the past half century, our preoccupation with epidemics of a biological nature has lessened while our obsession with epidemics of a social and technological nature has dramatically increased. This has been accompanied by a growing realisation that many of the epidemiological techniques used in the modelling of biological diseases can be readily transplanted into social and technological domains such as content and information diffusion, rumour spreading, gossiping protocols and viral marketing.

There is one recent but crucial respect in which our conceptual understanding of biological epidemics has advanced dramatically. In particular, it has become increasingly realised that it is important to study the interplay between 
pathogenic agents and between pathogenic agents and their environment. The corresponding field of study is known as synepidemiology in which the subjects of study are syndemics and counter-syndemics [35]. A syndemic is a set of mutually reinforcing health problems whose combined impact is more devastating than sum of the health problems in isolation (e.g. the risk developing tuberculosis is estimated to be between 12-20 times high for people with HIV [21]), while a counter-syndemic concerns a set of mutually inhibiting health problems whose combined impact is not as high as the sum of the health problems in isolation (e.g. studies suggest that a measles infection can temporarily inhibit the replication of the HIV virus [27]). Very lately, there has been a growing awareness that syndemics may also exist in a technological context: e.g. the purchase of a smartphone may make the purchase of the corresponding accessories and applications more likely [30].

In this paper, we extend the well-known Susceptible-Infected-Recovered (SIR) compartmental epidemiological model to support the interplay of multiple interacting epidemics. Our focus is on a scenario of two potentially-interacting epidemics spreading across a set of overlapping subpopulations. In this context, we derive a Markov model which describes the state changes of an individual with respect to each epidemic and whose transition rates incorporate syndemic and counter-syndemic interactions. The fluid limit of this Markov model reduces to a set of coupled SIR-type ODEs, the solution of which describes the evolution of the number of individuals infected by each epidemic.

The remainder of this paper is organised as follows. Section 2 presents an historical perspective on conceptual frameworks and modelling efforts pertinent to the field of epidemic modelling in the biological, social and technological domains. Section 3 presents our approach in extending the SIR model to support interacting epidemics, while Section 4 presents case studies of two interacting SIR epidemics propagating through two intersecting populations with various degrees of overlap. Section 5 concludes and considers avenues for future work.

\section{Background}

Human societies have been ravaged by biological epidemics throughout history with recurrent deadly outbreaks of bubonic plague, smallpox, yellow fever, cholera and influenza [38]. As shown in Fig. 1, the predominant early theories of disease causation were mostly supernatural, astronomical or religious, with causal agents including evil spirits, planetary motion and divine retribution. From the Middle Ages until Victorian times, it was also believed that if one inhaled miasmas - toxic vapors that emanated from swamps or decaying organic matter - disease would result [32]. Progress towards a more scientific and data-based approach began to be made from 1600 onwards with the collection of the first public health statistics, by John Graunt (1620-1674) [11] and others. One of the most famous studies was by John Snow of the 1854 London Cholera epidemic [36] in which he identified a particular water pump as the likely source of the outbreak. 


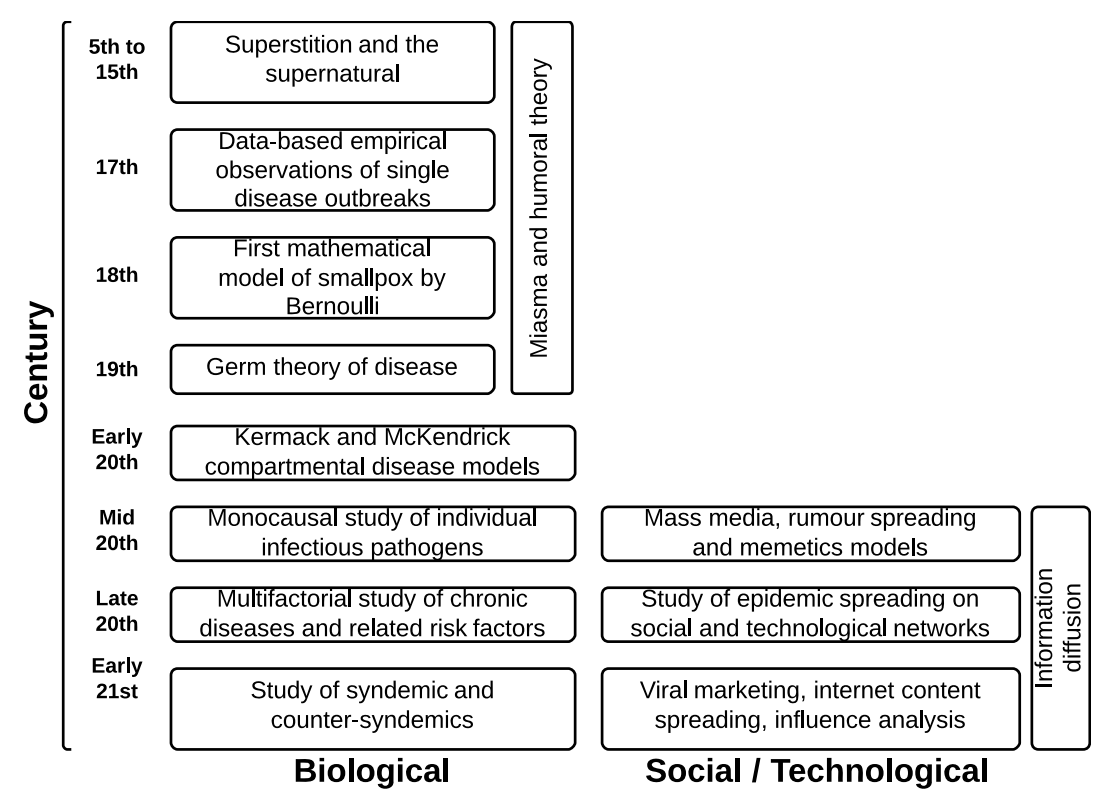

Fig. 1. The historical development of conceptual frameworks for epidemics

Predictive mathematical models for epidemics were relatively slow to develop, despite their utility in understanding, managing and forecasting epidemics. One of the earliest was by Daniel Bernoulli who carried out a study of the effects of smallpox vaccination in 1766 [9]. But arguably the most significant breakthrough came with the compartmental disease models proposed by Kermack and McKendrick in 1927 [19]. These elegantly express disease dynamics as coupled ODEs. The most well-known model is the Susceptible-Infected-Recovered (SIR) model. SIR features a closed population of individuals divided into three evolving subpopulations: $S(t)$ tracks those susceptible to become infected by the disease at time $t, I(t)$ tracks those infected by the disease with rate $\beta$ and $R(t)$ tracks those who have recovered from the disease at rate $\gamma$.

The science of epidemiology has made rapid advances in recent times and has moved from monocausal studies of infectious diseases to multifactor studies of chronic diseases (e.g. obesity). It has become increasingly realised that many diseases feature a complex web of interconnected risk factors (the so-called web of causation), which may include relationships with other diseases and relationships between diseases and the environment. The latter point of view is central to the science of synepidemiology [12]. Related mathematical models have been concurrently evolving, with some studies of the dynamics of two possibly-dependent co-infections in single populations $[4,25]$.

Of course it is not only disease which spreads in an epidemic fashion and researchers have proved adept at progressively transplanting the corresponding theory into sociological and technological domains, especially those related to in- 
formation diffusion. In the middle of the 20th century, spreading-process models for rumours, ideas and memes were proposed for the first time $[5,7,13]$, followed by mathematical models of how information spreads under mass media dissemination $[17,23]$. Various networks have subsequently come under the spotlight including computer networks [14], vehicular networks [39], mobile and ad-hoc networks [20], peer-to-peer file-sharing networks [22], mobile networks [34], wireless sensor networks $[2,6]$ and social networks $[8,16]$. More recently, mathematical models were developed to yield insights into the dynamics of emerging infectious diseases from social and technological network data $[3,15,18,28,29,31]$. There have also been studies analysing how user behaviour varies within user communities defined by a recommendation network [24], which creates viral marketing effects as well as studies about the role of centrality and influence in information diffusion within social networks $[1,26,33,37]$.

\section{Epidemic Model}

We focus on two interacting SIR (susceptible, infected, recovered) processes living on a finite set of overlapping subpopulations $P_{i}$ constituting a population $P=\cup_{i} P_{i}$. For notational convenience, we introduce the partition $\mathcal{P}$ of the population $P$ induced by the overlapping sub-populations. For each part $p$ in the partition, let its neighbourhood $\mathcal{N}(p)$ be a set of parts which includes $p$. Moreover, the size of the population of part $p$ is denoted by $n(p)$.

Remark 1. The neighbourhood of any part will be used to relate an individual's view-of-the-world to its infection rate. To make this concrete, consider a simple example where there are two subpopulations with a non-empty intersection. These overlapping subpopulations induce a partition with 3 parts: the two parts of individuals that belong to one subpopulation and not to the other, and the part corresponding to the intersection. As individuals in the intersection belong to both sub-populations, their neighbourhood includes all parts. The individuals that only belong to a single sub-population only see their own sub-population. Their neighbourhood therefore consists of their own part and the intersection.

Any individual of the population is susceptible to, infected by or recovered from any of two epidemics. The state of an individual is described by a pair $(k, \ell)$, with $k, \ell \in\{s, i, r\}$, where $s, i$ and $r$ stand for susceptible, infected and recovered, respectively and where $k$ and $\ell$ refer to the first and second epidemic, respectively. We consider a Markovian epidemic model and its fluid limit. At any point in time, the state of the Markov chain is described by the number of individuals in the different states and in the different parts.

Prior to introducing the Markov chain, some additional notation is required. Let $x_{(k, \ell)}^{p}$ be the number of individuals of part $p$ that are in state $(k, \ell)$, and let $\mathrm{x}$ be the vector with elements $x_{(k, \ell)}^{p}$, for $p \in \mathcal{P}$ and $k, l \in\{s, i, r\}$. The state space $\mathcal{X}$ of the Markov chain is defined as the set of vectors $\mathbf{x}$ such that,

$$
x_{(k, \ell)}^{p} \in \mathbb{N}=\{0,1,2, \ldots\}, \sum_{k, \ell \in\{s, i, r\}} x_{(k, \ell)}^{p}=n(p) \quad \text { for all } p \in \mathcal{P} .
$$




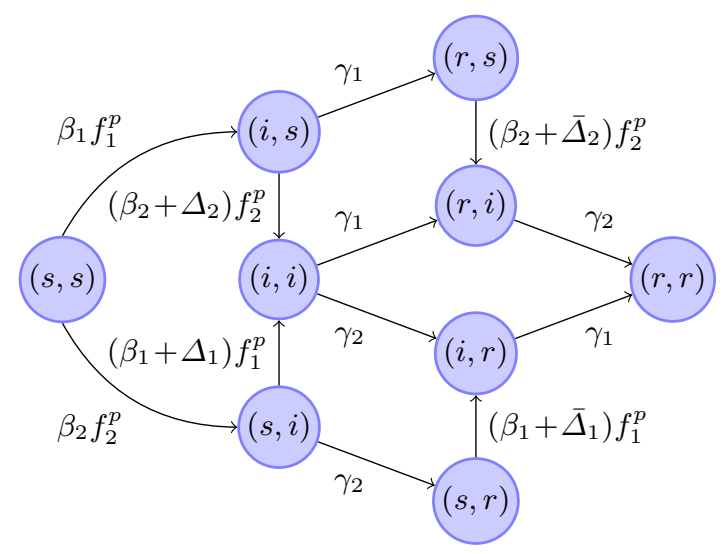

Fig. 2. Transition rates for an individual in part $p$.

Moreover, let $\mathbf{e}_{(k, \ell)}^{p}$, for $p \in \mathcal{P}$ and $k, l \in\{s, i, r\}$ be the obvious unit vectors of the state space $\mathcal{X}$. The following parameters describe the transition rates for changing states. An $(s, s)$-individual in part $p$ gets infected by the first and second epidemics with rates $\beta_{1} f_{1}^{p}(\mathbf{x})$ and $\beta_{2} f_{2}^{p}(\mathbf{x})$, respectively. Here, $f_{1}^{p}(\mathbf{x})$ and $f_{1}^{p}(\mathbf{x})$ are the fractions of individuals that are infected by epidemic 1 and 2 in the neighbourhood of $p \in \mathcal{P}$,

$$
\begin{aligned}
f_{1}^{p}(\mathbf{x}) & =\frac{\sum_{q \in \mathcal{N}(p)}\left(x_{(i, r)}^{q}+x_{(i, s)}^{q}+x_{(i, i)}^{q}\right)}{\sum_{q \in \mathcal{N}(p)} n(q)}, \\
f_{2}^{p}(\mathbf{x}) & =\frac{\sum_{q \in \mathcal{N}(p)}\left(x_{(r, i)}^{q}+x_{(s, i)}^{q}+x_{(i, i)}^{q}\right)}{\sum_{q \in \mathcal{N}(p)} n(q)} .
\end{aligned}
$$

If such an individual is already infected by or has already recovered from the other epidemic, the infection rate is modified. An $(s, i)$ individual in part $p$ gets infected by the first epidemic with rate $\left(\beta_{1}+\Delta_{1}\right) f_{1}^{p}(\mathbf{x})$, while an $(s, r)$ individual gets infected by the first epidemic with rate $\left(\beta_{1}+\bar{\Delta}_{1}\right) f_{1}^{p}(\mathbf{x})$. Modified infection rates are defined likewise for the second epidemic. Finally, the recovery rates of an individual from epidemic 1 and 2 are constant and equal to $\gamma_{1}$ and $\gamma_{2}$, respectively. For clarity, the transition rates for an individual in part $p$ are depicted in Figure 2. The infinitesimal generator $\mathcal{A}$ of this Markov chain is:

$$
\begin{aligned}
\mathcal{A} g(\mathbf{x})=\sum_{p \in \mathcal{P}}\left(\beta_{1}\right. & f_{1}^{p}(\mathbf{x}) x_{(s, s)}^{p}\left[g\left(\mathbf{x}-\mathbf{e}_{(s, s)}^{p}+\mathbf{e}_{(i, s)}^{p}\right)-g(\mathbf{x})\right] \\
& +\beta_{2} f_{2}^{p}(\mathbf{x}) x_{(s, s)}^{p}\left[g\left(\mathbf{x}-\mathbf{e}_{(s, s)}^{p}+\mathbf{e}_{(s, i)}^{p}\right)-g(\mathbf{x})\right] \\
& +\left(\beta_{1}+\Delta_{1}\right) f_{1}^{p}(\mathbf{x}) x_{(s, i)}^{p}\left[g\left(\mathbf{x}-\mathbf{e}_{(s, i)}^{p}+\mathbf{e}_{(i, i)}^{p}\right)-g(\mathbf{x})\right] \\
& +\left(\beta_{2}+\Delta_{2}\right) f_{2}^{p}(\mathbf{x}) x_{(i, s)}^{p}\left[g\left(\mathbf{x}-\mathbf{e}_{(i, s)}^{p}+\mathbf{e}_{(i, i)}^{p}\right)-g(\mathbf{x})\right]
\end{aligned}
$$




$$
\begin{aligned}
& +\left(\beta_{1}+\bar{\Delta}_{1}\right) f_{1}^{p}(\mathbf{x}) x_{(s, r)}^{p}\left[g\left(\mathbf{x}-\mathbf{e}_{(s, r)}^{p}+\mathbf{e}_{(i, r)}^{p}\right)-g(\mathbf{x})\right] \\
& +\left(\beta_{2}+\bar{\Delta}_{2}\right) f_{2}^{p}(\mathbf{x}) x_{(r, s)}^{p}\left[g\left(\mathbf{x}-\mathbf{e}_{(r, s)}^{p}+\mathbf{e}_{(r, i)}^{p}\right)-g(\mathbf{x})\right] \\
& +\gamma_{1} x_{(i, s)}^{p}\left[g\left(\mathbf{x}-\mathbf{e}_{(i, s)}^{p}+\mathbf{e}_{(r, s)}^{p}\right)-g(\mathbf{x})\right] \\
& +\gamma_{1} x_{(i, i)}^{p}\left[g\left(\mathbf{x}-\mathbf{e}_{(i, i)}^{p}+\mathbf{e}_{(r, i)}^{p}\right)-g(\mathbf{x})\right] \\
& +\gamma_{1} x_{(i, r)}^{p}\left[g\left(\mathbf{x}-\mathbf{e}_{(i, r)}^{p}+\mathbf{e}_{(r, r)}^{p}\right)-g(\mathbf{x})\right] \\
& +\gamma_{2} x_{(s, i)}^{p}\left[g\left(\mathbf{x}-\mathbf{e}_{(s, i)}^{p}+\mathbf{e}_{(s, r)}^{p}\right)-g(\mathbf{x})\right] \\
& +\gamma_{2} x_{(i, i)}^{p}\left[g\left(\mathbf{x}-\mathbf{e}_{(i, i)}^{p}+\mathbf{e}_{(i, r)}^{p}\right)-g(\mathbf{x})\right] \\
& \left.+\gamma_{2} x_{(r, i)}^{p}\left[g\left(\mathbf{x}-\mathbf{e}_{(r, i)}^{p}+\mathbf{e}_{(r, r)}^{p}\right)-g(\mathbf{x})\right]\right)
\end{aligned}
$$

for $\mathrm{x} \in \mathcal{X}$. Due to the considerable size of the state space $\mathcal{X}$, even for modest population sizes, direct computation of either transient or stationary distributions is quite forbidding. As we are mainly interested in the dynamics when the population is large, we focus on the fluid limit of the process. However, the original Markov chain will also be simulated and compared with the fluid limits.

More specifically, we consider a sequence of Markov chains with generators $\mathcal{A}_{N}$ such that the population size is $N$ for the $N$ th Markov chain and we keep track of the fractions of populations, such that components of the state space $\mathcal{X}_{N}$ of the $N$ th Markov chain live on a lattice with step size $1 / N$, and the unit vectors have size $1 / N$ as well. By contrast, the transition rates increase by $N$ as we need to translate from population fractions to population sizes. Setting $\epsilon:=1 / N$, we get the following generator:

$$
\begin{aligned}
\mathcal{A}_{\epsilon^{-1}} g(\mathbf{x})=\epsilon^{-1} \sum_{p \in \mathcal{P}}( & \beta_{1} f_{1}^{p}(\mathbf{x}) x_{(s, s)}^{p}\left[g\left(\mathbf{x}-\epsilon \mathbf{e}_{(s, s)}^{p}+\epsilon \mathbf{e}_{(i, s)}^{p}\right)-g(\mathbf{x})\right] \\
& +\beta_{2} f_{2}^{p}(\mathbf{x}) x_{(s, s)}^{p}\left[g\left(\mathbf{x}-\epsilon \mathbf{e}_{(s, s)}^{p}+\epsilon \mathbf{e}_{(s, i)}^{p}\right)-g(\mathbf{x})\right] \\
& +\left(\beta_{1}+\Delta_{1}\right) f_{1}^{p}(\mathbf{x}) x_{(s, i)}^{p}\left[g\left(\mathbf{x}-\epsilon \mathbf{e}_{(s, i)}^{p}+\epsilon \mathbf{e}_{(i, i)}^{p}\right)-g(\mathbf{x})\right] \\
& +\left(\beta_{2}+\Delta_{2}\right) f_{2}^{p}(\mathbf{x}) x_{(i, s)}^{p}\left[g\left(\mathbf{x}-\epsilon \mathbf{e}_{(i, s)}^{p}+\epsilon \mathbf{e}_{(i, i)}^{p}\right)-g(\mathbf{x})\right] \\
& +\left(\beta_{1}+\bar{\Delta}_{1}\right) f_{1}^{p}(\mathbf{x}) x_{(s, r)}^{p}\left[g\left(\mathbf{x}-\epsilon \mathbf{e}_{(s, r)}^{p}+\epsilon \mathbf{e}_{(i, r)}^{p}\right)-g(\mathbf{x})\right] \\
& +\left(\beta_{2}+\bar{\Delta}_{2}\right) f_{2}^{p}(\mathbf{x}) x_{(r, s)}^{p}\left[g\left(\mathbf{x}-\epsilon \mathbf{e}_{(r, s)}^{p}+\epsilon \mathbf{e}_{(r, i)}^{p}\right)-g(\mathbf{x})\right] \\
& +\gamma_{1} x_{(i, s)}^{p}\left[g\left(\mathbf{x}-\epsilon \mathbf{e}_{(i, s)}^{p}+\epsilon \mathbf{e}_{(r, s)}^{p}\right)-g(\mathbf{x})\right] \\
& +\gamma_{1} x_{(i, i)}^{p}\left[g\left(\mathbf{x}-\epsilon \mathbf{e}_{(i, i)}^{p}+\epsilon \mathbf{e}_{(r, i)}^{p}\right)-g(\mathbf{x})\right] \\
& +\gamma_{1} x_{(i, r)}^{p}\left[g\left(\mathbf{x}-\epsilon \mathbf{e}_{(i, r)}^{p}+\epsilon \mathbf{e}_{(r, r)}^{p}\right)-g(\mathbf{x})\right] \\
& +\gamma_{2} x_{(s, i)}^{p}\left[g\left(\mathbf{x}-\epsilon \mathbf{e}_{(s, i)}^{p}+\epsilon \mathbf{e}_{(s, r)}^{p}\right)-g(\mathbf{x})\right] \\
& +\gamma_{2} x_{(i, i)}^{p}\left[g\left(\mathbf{x}-\epsilon \mathbf{e}_{(i, i)}^{p}+\epsilon \mathbf{e}_{(i, r)}^{p}\right)-g(\mathbf{x})\right] \\
& \left.+\gamma_{2} x_{(r, i)}^{p}\left[g\left(\mathbf{x}-\epsilon \mathbf{e}_{(r, i)}^{p}+\epsilon \mathbf{e}_{(r, r)}^{p}\right)-g(\mathbf{x})\right]\right) .
\end{aligned}
$$

We can deduce the (candidate) fluid limit by Taylor expansion of this generator around $\epsilon=0$, from which we find a limiting generator of the form 
$\hat{\mathcal{A}} g=\mathbf{h}(\mathbf{x}) \cdot \nabla g$, for a certain $9|\mathcal{P}|$-dimensional vector function $\mathbf{h}$. Note that a generator of this form corresponds to a deterministic process satisfying the system of differential equations $\dot{\mathbf{x}}(t)=\mathbf{h}(\mathbf{x}(t))$.

In order to prove this limit rigourously, it needs to be checked that both the pre-limit processes and the limit process are Feller processes [10], which basically boils down to checking the so-called Hille-Yosida conditions. We believe that a careful proof of this statement falls outside the scope of this paper, but remark that due to the compactness of the state space (in the prelimit as well as in the limit), the proof is not as involved as is sometimes the case. Below we detail the set of differential equations, where we have dropped the dependence of $t$ for notational convenience.

After some manipulations we find the following fluid limit which not only generalises syndemics in a single population but also epidemics on a stratified population:

$$
\begin{aligned}
\dot{x}_{(s, s)}^{p} & =-\beta_{1} y_{1}^{p} x_{(s, s)}^{p}-\beta_{2} y_{2}^{p} x_{(s, s)}^{p} \\
\dot{x}_{(i, s)}^{p} & =\beta_{1} y_{1}^{p} x_{(s, s)}^{p}-\left(\beta_{2}+\Delta_{2}\right) y_{2}^{p} x_{(i, s)}^{p}-\gamma_{1} x_{(i, s)}^{p} \\
\dot{x}_{(s, i)}^{p} & =\beta_{2} y_{2}^{p} x_{(s, s)}^{p}-\left(\beta_{1}+\Delta_{1}\right) y_{1}^{p} x_{(s, i)}^{p}-\gamma_{2} x_{(s, i)}^{p} \\
\dot{x}_{(i, i)}^{p} & =\left(\beta_{2}+\Delta_{2}\right) y_{2}^{p} x_{(i, s)}^{p}+\left(\beta_{1}+\Delta_{1}\right) y_{1}^{p} x_{(s, i)}^{p}-\left(\gamma_{1}+\gamma_{2}\right) x_{(i, i)}^{p} \\
\dot{x}_{(r, s)}^{p} & =\gamma_{1} x_{(i, s)}^{p}-\left(\beta_{2}+\bar{\Delta}_{2}\right) y_{2}^{p} x_{(r, s)}^{p} \\
\dot{x}_{(r, i)}^{p} & =\left(\beta_{2}+\bar{\Delta}_{2}\right) y_{2}^{p} x_{(r, s)}^{p}+\gamma_{1} x_{(i, i)}^{p}-\gamma_{2} x_{(r, i)}^{p} \\
\dot{x}_{(i, r)}^{p} & =\left(\beta_{1}+\bar{\Delta}_{1}\right) y_{1}^{p} x_{(s, r)}^{p}+\gamma_{2} x_{(i, i)}^{p}-\gamma_{1} x_{(i, r)}^{p} \\
\dot{x}_{(s, r)}^{p} & =\gamma_{2} x_{(s, i)}^{p}-\left(\beta_{1}+\bar{\Delta}_{1}\right) y_{1}^{p} x_{(s, r)}^{p} \\
\dot{x}_{(r, r)}^{p} & =\gamma_{1} x_{(i, r)}^{p}+\gamma_{2} x_{(r, i)}^{p} \\
y_{1}^{p}= & \frac{\sum_{q \in \mathcal{N}(p)}\left(x_{(i, s)}^{q}+x_{(i, i)}^{q}+x_{(i, r)}^{q}\right)}{\sum_{q \in \mathcal{N}(p)} \nu(p)} \\
y_{2}^{p}= & \frac{\sum_{q \in \mathcal{N}(p)}\left(x_{(s, i)}^{q}+x_{(i, i)}^{q}+x_{(r, i)}^{q}\right)}{\sum_{q \in \mathcal{N}(p)} \nu(q)}
\end{aligned}
$$

for $p \in \mathcal{P}$. The fractions $y_{1}^{p}$ and $y_{2}^{p}$ were introduced in the set of ODEs to simplify notation: $y_{i}^{p}(t)$ is the fraction of individuals that are infected by epidemic $i$ in the neighbourhood of $p$.

\section{Case Studies}

With the ODEs established we now focus on some numerical examples. To limit the number of parameters, we investigate the spread of two epidemics, say $e_{1}$ and $e_{2}$, on two intersecting populations. For both epidemics, the spreading and recovery parameters are set to $\beta_{i}=0.4$ and $\gamma_{i}=0.1(i=1,2)$, respectively. 
There are two populations. Population $P 1$ constitutes $30 \%$ of the total population. The population $P 2$ constitutes $70 \%$ of the total population. The fraction of the individuals in the intersection of both populations - referred to as the degree of overlap - is denoted by $\nu$ and assumed to be $0.01 \%$ unless indicated otherwise. For a fixed $\nu, 30 \%-\nu / 2$ and $70 \%-\nu / 2$ of the individuals are in $P 1$ and not in $P 2$ and in $P 2$ and not in $P 1$, respectively.

For all case studies $\bar{\Delta}_{1}=\Delta_{1}$ and $\bar{\Delta}_{2}=\Delta_{2}$. Epidemic $e_{1}$ begins in the non-intersecting population $P 1$ at time 0 , and epidemic $e_{2}$ begins in the nonintersecting population $P 2$ at time 0 . The initial number of infected individuals is $1 \%$ for each epidemic, and no individuals are infected by both epidemics at the start. With the parameters fixed, we now investigate how spreading of the epidemics is affected by (i) the size of the intersection, (ii) syndemic effects and (iii) counter-syndemic effects.

Case Study 1: Influence of Degree of Overlap Fig. 3 shows the influence of the degree of overlap $\nu$ between the populations on the spread of $e_{1}$ and $e_{2}$. We see that the smaller the intersection, the more significant the delay of the propagation of the epidemics between the populations. With values of $\nu$ above $1 \%$, the results are increasingly indistinguishable from epidemics spreading in a single population. The multimodality of the spread over time is quite apparent. The epidemics first reach their peak in the population in which they originated. Only after sufficiently many individuals in the intersection are affected, spreading in the other population starts, reaching its peak considerably later, even though the spreading mechanism is exactly the same in both populations and for both epidemics. Finally note that the first peak of $e_{2}$ is considerably higher than the first peak of $e_{1}$ while the opposite is observed for the second peak which is in line with the sizes of the populations the epidemics originate from.

Case Study 2: The Impact of Syndemic Effects Fig. 4 shows how syndemic effects affect the evolution of the epidemics. We consider three cases. For $\Delta_{1}=$ $\beta=0.4$ and $\Delta_{2}=0$, the second epidemic reinforces spreading of the first. Specifically, if an individual is infected by the second epidemic, its infection rate for the first epidemic is doubled. For $\Delta_{2}=\beta=0.4$ and $\Delta_{1}=0$, the first epidemic reinforces spreading of the second in a similar manner. Finally, for $\Delta_{1}=\Delta_{2}=$ $\beta=0.4$, both epidemics reinforce each other. For $\Delta_{1}=0, \Delta_{2}=0.4$ for $e_{1}$ corresponds to the case where there are no syndemic effects on $e_{1}$. Comparison with the other $e_{1}$ curves clearly reveals the syndemic effects. Particularly note that when both epidemics reinforce each other, the peak of $e_{1}$ is sooner and a little higher. This is explained by the fact that $e_{1}$ affects the spread of $e_{2}$ which in turn reinforces the spread of $e_{1}$. Similar observations apply to $e_{2}$.

Case study 3: The Impact of Counter-syndemic Effects Fig. 5 shows the impact of counter-syndemic effects. We consider three cases. For $\Delta_{1}=-\beta=$ -0.4 and $\Delta_{2}=0$, an individual infected by the second epidemic is immune to the 

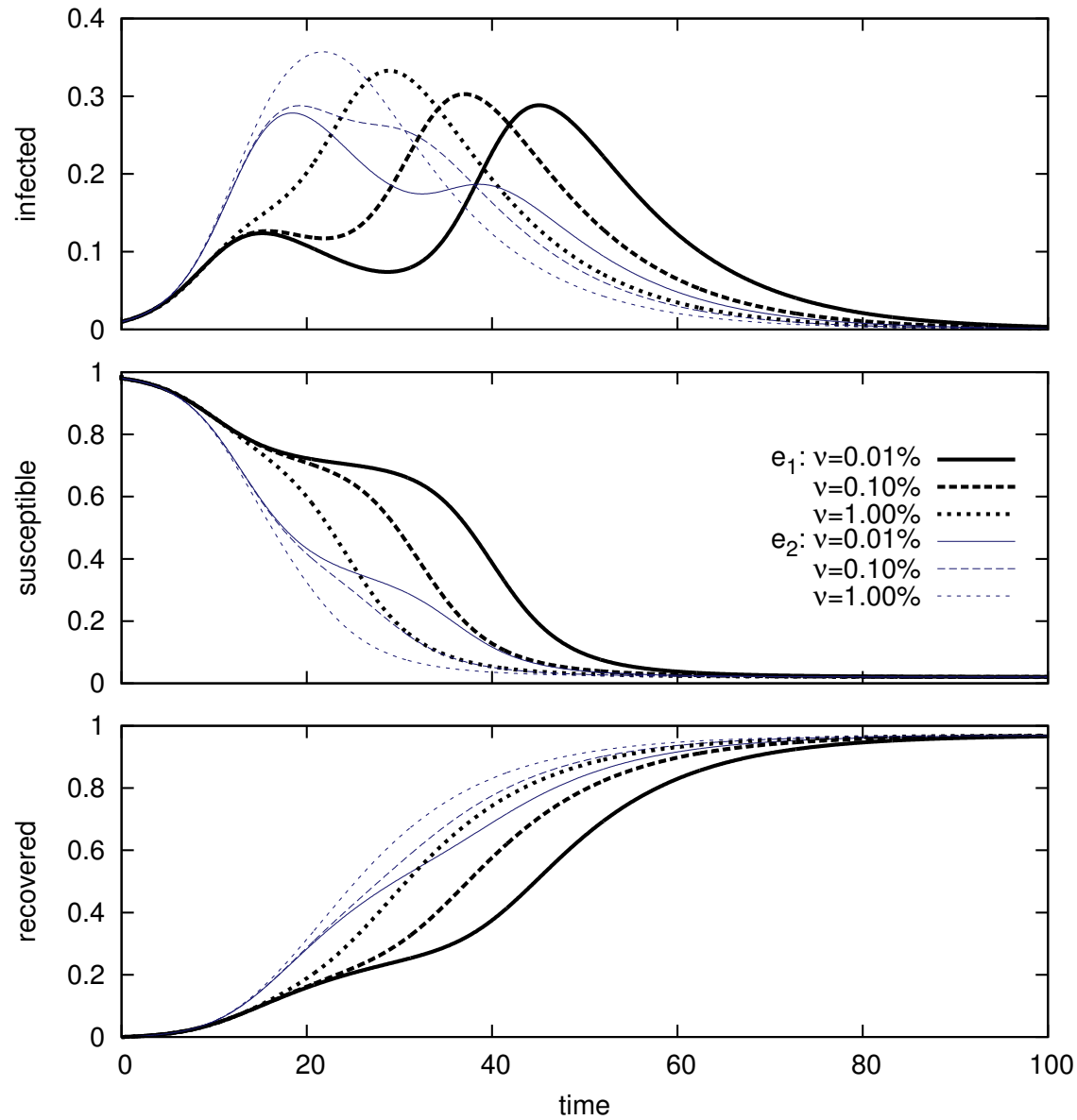

Fig. 3. Evolution of the fractions of infected, susceptible and recovered individuals for epidemics $e_{1}$ and $e_{2}$ and for different sizes of the intersection $\nu$ as indicated. 

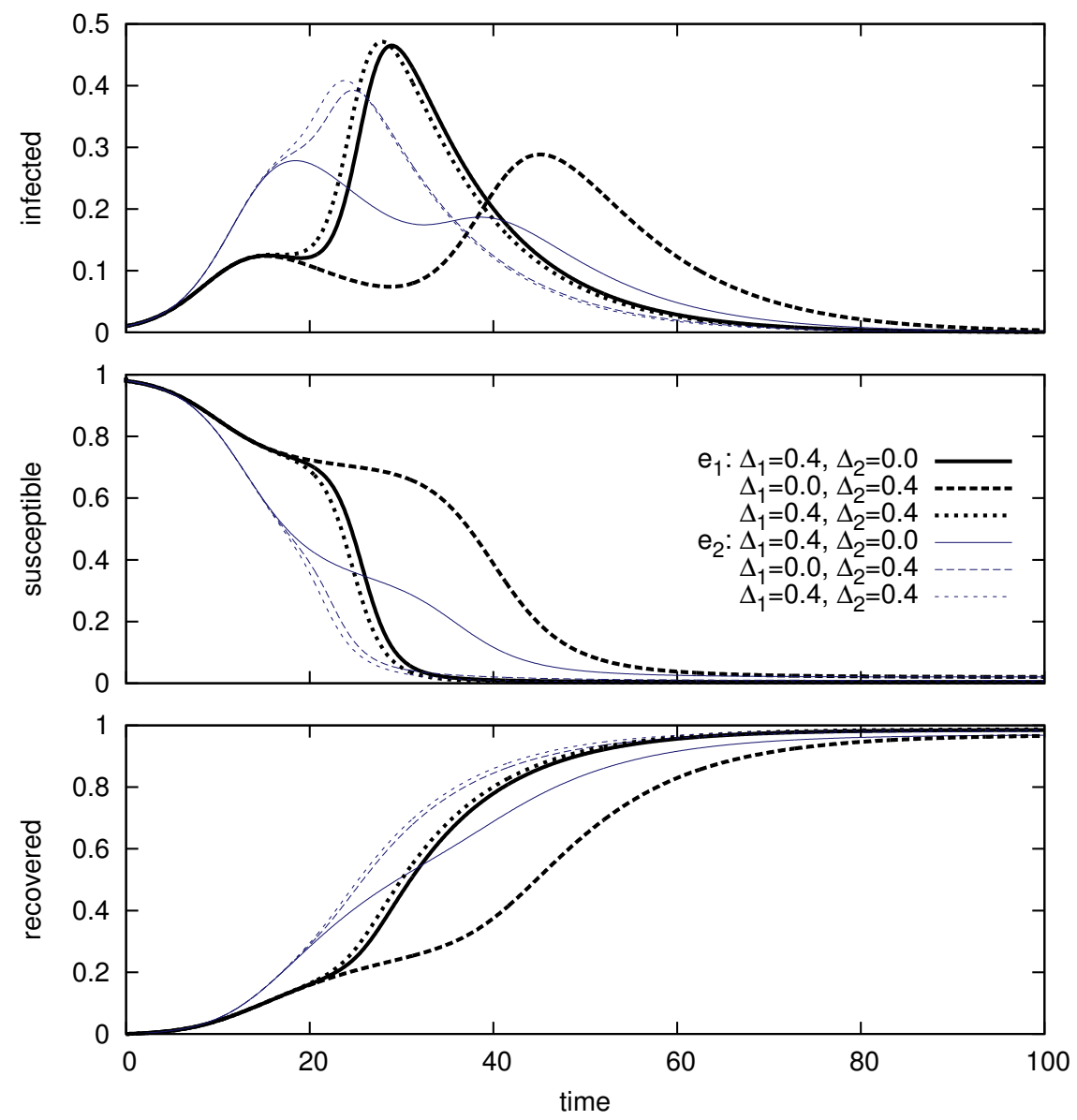

Fig. 4. Syndemic effects on the evolution of epidemics $e_{1}$ and $e_{2}$. 

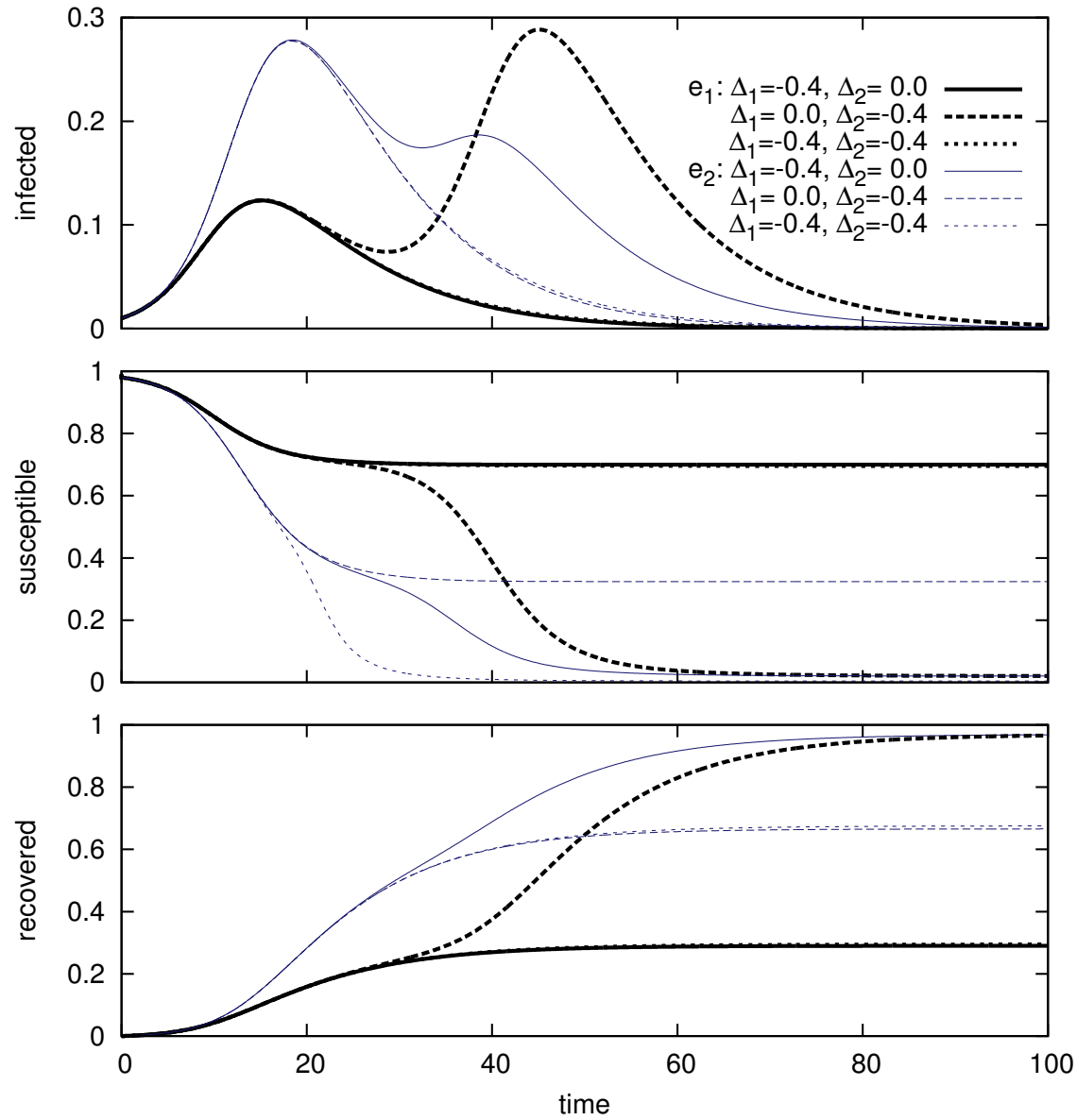

Fig. 5. Counter-syndemic effects on the evolution of epidemics $e_{1}$ and $e_{2}$. 
first epidemic. For $\Delta_{2}=-\beta=-0.4$ and $\Delta_{1}=0$, an individual infected by the first epidemic is immune to the second epidemic. Finally, for $\Delta_{1}=\Delta_{2}=-\beta=$ -0.4 , immunity works both ways. As similar effects apply for both epidemics, we focus on $e_{1}$. Clearly, for $\Delta_{2}=-\beta=-0.4$ and $\Delta_{1}=0$, the first epidemic is not affected by syndemic effects. Hence, the $e_{1}$ curve for $\Delta_{2}=-\beta=-0.4$ and $\Delta_{1}=$ 0 can be used as reference. Comparing this curve with the other $e_{1}$ curves clearly illustrates counter-syndemic effects. In fact, the second peak of the epidemic is no longer present. This is explained by noting that this peak was reached in the population where the second epidemic originates. By the time the first epidemic reaches this population, most of its individuals are already immune. Finally, note that a large proportion of the population remains susceptible to the first epidemic.

\section{Conclusion}

It is important that the sophistication of mathematical modelling techniques keeps pace with our evolving understanding of the dynamics of epidemic processes, especially as they become applied in myriad domains beyond the biological. Our present paper has made some progress in this direction by considering models of syndemic and counter-syndemic interactions between two SIR epidemics in multiple overlapping populations. The results from this kind of analysis can give insights into epidemic forecasting and optimal strategies for managing the response to outbreaks.

Much more remains to be done. For example, while the present work targets fluid limits, other scalings leading to diffusion limits may shed light on the variance of outcomes. In addition, our populations are assumed to be static when a more realistic model might assume some dynamic movement of individuals between populations (practically realised as facilities to join and leave populations). Practical case studies could also be carried out in application areas ranging from computer viruses to extreme ideologies.

\section{References}

1. K. Avrachenkov, K. De Turck, D. Fiems, and B. J. Prabhu. Information dissemination processes in directed social networks. CoRR, abs/1311.2023, 2013.

2. A. Boukerche. Epidemic Models, Algorithms, and Protocols in Wireless Sensor and Ad Hoc Networks. Wiley-IEEE Press, 2008.

3. M. Cha, H. Kwak, P. Rodriguez, Y-Y. Ahn, and S. Moon. I tube, you tube, everybody tubes: analyzing the world's largest user generated content video system. In IMC '07: Proc. 7th ACM SIGCOMM, pages 1-14, NY, USA, 2007. ACM.

4. L. Chen, F. Ghanbarnejad, W. Cai, and P. Grassberger. Outbreaks of coinfections: The critical role of cooperativity. Europhysics Letters, 104, December 2013.

5. D. J. Daley and D. G. Kendall. Epidemics and rumours. Nature, 204:1118, 1964.

6. A. Datta, S. Quarteroni, and K. Aberer. Autonomous gossiping: A self-organizing epidemic algorithm for selective information dissemination in wireless mobile adhoc networks. In Semantics of a Networked World. Semantics for Grid Databases, 
volume 3226 of Lecture Notes in Computer Science, pages 126-143. Springer Berlin Heidelberg, 2004.

7. R. Dawkins. The Selfish Gene. Oxford University Press, Oxford, UK, 1976.

8. E. De Cuypere, K. De Turck, S. Wittevrongel, and D. Fiems. Markovian sir model for opinion propagation. In Proceedings of the 2013 25th International Teletraffic Congress (ITC), pages 1-7. IEEE, 2013.

9. K. Dietz and J. A. P. Heesterbeek. Daniel Bernoulli's epidemiological model revisited. Mathematical Biosciences, 180:1-21, 2002.

10. S. N. Ethier and T. G. Kurtz. Markov processes - characterization and convergence. Wiley Series in Probability and Mathematical Statistics: Probability and Mathematical Statistics. John Wiley \& Sons Inc., New York, 1986.

11. J. Gaunt. Natural and Political Observations Mentioned in a following index, and made upon the Bills of Mortality. 1662. Available online at http://www . neonatology. org/pdf/graunt.pdf.

12. L. Getz. Sustainable and responsible preventive medicine: Conceptualising ethical dilemmas arising from clinical implementation of advancing medical technology. $\mathrm{PhD}$ thesis, Norweigan University of Science and Technology, Trondheim, 2006.

13. W. Goffman and V. A. Newill. Generalization of epidemic theory: An application to the transmission of ideas. Nature, 204:225-228, October 1964.

14. S. M. Hedetniemi, S. T. Hedetniemi, and A. L. Liestman. A survey of gossiping and broadcasting in communication networks. Networks, 18(4):319-349, 1988.

15. H.-W. Hu and S.-Y. Lee. Study on influence diffusion in social network. International Journal of Computer Science and Electronics Engineering (IJCSEE), 1, 2013.

16. J. L. Iribarren and E. Moro. Information diffusion epidemics in social networks. Physical Review, 2009.

17. Y. Jaewon and J. Leskovec. Modeling information diffusion in implicit networks. In Data Mining (ICDM), 2010 IEEE 10th International Conference on, pages 599 608, Dec 2010.

18. A. Karnik, A. Saroop, and V. Borkar. On the diffusion of messages in on-line social networks. Performance Evaluation, 70(4):271 - 285, 2013.

19. W. O. Kermack and A. G. McKendrick. Contributions to the mathematical theory of epidemics-I. 1927. Bull Math Biol, 53(1-2):33-55, 1991.

20. A. Khelil, C. Becker, J. Tian, and K. Rothermel. An epidemic model for information diffusion in MANETs. In Proc. 5th ACM International Workshop on Modeling Analysis and Simulation of Wireless and Mobile Systems, pages 54-60, 2002.

21. C. Kwan and J. Ernst. HIV and tuberculosis: a deadly human syndemic. Clinical Microbiology, 24(2):351-376, 2011.

22. K. Leibnitz, T. Hobfeld, N. Wakamiya, and M. Murata. Modeling of epidemic diffusion in peer-to-peer file-sharing networks. In The Second International Workshop on Biologically Inspired Approaches to Advanced Information Technology, Bio-ADIT 2006, LNCS 3853, Osaka, Japan, 2006.

23. K. Lerman and R. Ghosh. Information contagion: An empirical study of the spread of news on Digg and Twitter social networks. Proc. 4th International AAAI Conference on Weblogs and Social Media (ICWSM 2010), 2010.

24. J. Leskovec, L. A. Adamic, and B. A. Huberman. The dynamics of viral marketing. ACM Trans. Web, 1(1), May 2007.

25. M. Martcheva and S.S. Pilyugin. The role of coinfection in multi-disease dynamics. SIAM Journal of Applied Mathematics, 66:843-872, 2006.

26. A. Mochalova and A. Nanopoulos. On the role of centrality in information diffusion in social networks. In Proc. ECIS 2013, page 101, 2013. 
27. W. J. Moss, S. Scott, Z. Ndhlovu, M. Monze, F. T. Cutts, T. C. Quinn, and D.E. Griffin. Suppression of human immunodeficiency virus type 1 viral load during acute measles. Pediatric Infectious Disease Journal, 28(1):63-65, 2009.

28. S. Myers and J. Leskovec. Clash of the Contagions: Cooperation and Competition in Information Diffusion. In Proc. IEEE International Conference on Data Mining (ICDM 2012), 2012.

29. P. Netrapalli and S. Sanghavi. Learning the graph of epidemic cascades. In Proc. 12th ACM SIGMETRICS/PERFORMANCE Joint International Conference on Measurement and Modeling of Computer Systems (SIGMETRICS 2012), pages 211-222, New York, NY, USA, 2012. ACM.

30. M. E. J. Newman and C. R. Ferrario. Interacting epidemics and coinfection on contact networks. PLoS ONE, 8(8):e71321, 082013.

31. M. Nika, G. Ivanova, and W. J. Knottenbelt. On celebrity, epidemiology and the internet. In Proc. 7th International Conference on Performance Evaluation Methodologies and Tools (VALUETOOLS 2013), Turin, Italy, December 2013.

32. R. Parker. Miasma: Pollution and Purification in Early Greek Religion. Clarendon paperbacks. Clarendon Press, 1990.

33. S.-M. Pi, Y.-C. Liu, T.-Y. Chen, and S.-H. Li. The influence of instant messaging usage behavior on organizational communication satisfaction. In Proc. 41st Annual Hawaii International Conference on System Sciences, HICSS '08, page 449, Washington, DC, USA, 2008. IEEE Computer Society.

34. C. J. Rhodes and M. Nekovee. The opportunistic transmission of wireless worms between mobile devices. CoRR, abs/0802.2685, 2008.

35. M. Singer. Introduction to Syndemics. Wiley, 2009.

36. J. Snow. On the Mode of Communication of Cholera. John Churchill, 1855.

37. L. Weng, A. Flammini, A. Vespignani, and F. Menczer. Competition among memes in a world with limited attention. Scientific Reports, 2(335), 2013.

38. Wikipedia. List of epidemics. http://en.wikipedia.org/wiki/List_of_ epidemics.

39. L. Wischhof, A. Ebner, and H. Rohling. Information dissemination in selforganizing intervehicle networks. Intelligent Transportation Systems, IEEE Transactions on, 6(1):90-101, March 2005. 